\title{
Children's at Home: Pilot study assessing dedicated social media for parents of adolescents with Neurofibromatosis Type 1.
}

\section{ABSTRACT}

The aim of this pilot study was to evaluate Children's at Home (C@H), a dedicated social media website for parents of adolescents with Neurofibromatosis Type 1 (NF1).

The interventional study included 2 phases: (1) Creating Video Intervention/Prevention Assessment (VIA) visual narratives about having an adolescent with NF1; (2) Interacting on C@H, a secure, medicallymoderated social media website. C@H was evaluated qualitatively at 3 time points.

At enrollment (T0, N=17) - participants reported needing C@H to break their isolation, connect with other families, and receive accurate information, advice, and support from others facing similar challenges. At T1, after creating VIA during 6 months ( $N=13,145$ videos), participants mostly valued the opportunity to speak about the challenges they face with NF1 and their journey since diagnosis. At T2, after interacting on $\mathrm{C} @ \mathrm{H}$ for 7 weeks ( $\mathrm{N}=10,2$ sign-ins/week/parent), participants reported connecting with other parents of children with NF1 for the first time, valuing the "real faces" and emotions of other parents with shared experiences providing a sense of normalcy. Qualitative analysis suggested that C@H decreased feelings of isolation, provided relief to talk about NF1 without having to explain it, provided new knowledge about NF1 and the opportunity to address non-medical issues of NF1 never discussed in clinic, and helped participants with putting their lives into perspective.

C@H allowed parents of adolescents with NF1 to overcome previous isolation and connect for the first time. Innovative applications of social media dedicated to those who care for children with chronic conditions can provide peer-to-peer support, shared experience, and reliable medical information.

Keywords: Social media, peer support, adolescents, parents, intervention, neurofibromatosis 


\section{INTRODUCTION}

Parents of adolescents living with a chronic condition (CC) often feel isolated when facing challenges of adolescent development added to those imposed by the underlying condition (Akre \& Suris, 2014).

Parenting an adolescent with a CC is associated with increased levels of stress, distress, emotional problems, and depression (Brehaut et al., 2009; Brehaut et al., 2004; Frey, Greenberg, \& Fewell, 1989;

McKinney \& Peterson, 1987). When the child reaches adolescence, parents are commonly unprepared to deal with the developmental realities of this life-stage synergizing with the challenges linked to the CC. Studies have highlighted the importance parents attach to being able to share information and management strategies with peers living similar experiences (Akre, Ramelet, Berchtold, \& Suris, 2015; Akre \& Suris, 2014).

Neurofibromatosis type 1 (NF1) is an autosomal dominant disorder affecting approximately 1:3500 individuals, with diverse manifestations including neurocutaneous stigmata and a propensity to develop tumors of the central and peripheral nervous system (Ferner, 2007). More than half of individuals with NF1 experience cognitive impairment (Coude, Mignot, Lyonnet, \& Munnich, 2006; Hyman, Arthur Shores, \& North, 2006; Hyman, Shores, \& North, 2005; Levine, Materek, Abel, O'Donnell, \& Cutting, 2006), poorer social and emotional function (Barton \& North, 2004; Martin, Wolters, et al.), deficits in social skills and decreased success in academic and physical activities (Dilts et al., 1996; Johnson, Saal, Lovell, \& Schorry, 1999) compared to unaffected siblings and same-aged peers. Although little research has been conducted on the psychosocial aspects of living with NF1 for adolescents, there is growing evidence that these functional disability and illness-related variables have a negative impact on emotional quality of life and that cosmetic aspects of the underlying illness may result in anxiety (Benjamin et al., 1993; Garwood et al.; Graf, Landolt, Mori, \& Boltshauser, 2006). 
Parents of children with NF1 have been shown to experience considerable stress, doubt, and fears in caring for their children (Graf et al., 2006; Martin, Gillespie, Wolters, \& Widemann). Recent studies demonstrate the importance of evaluating the whole family in order to optimize the quality of life and psychological adjustment in children and adolescents with NF1 (Graf et al., 2006). Reiter-Purtill et al highlighted that mothers and fathers of children with NF1 experienced greater symptoms of distress when compared to parents of healthy controls (Reiter-Purtill et al., 2008). Parents play a fundamental role as an extension of the clinical care team. Because they live with and often manage children with a CC, parental experience with disease has been shown to have a profound impact on the child's wellbeing and quality of life (Graf et al., 2006).

Despite the major role parents play in their children's lives and development (Akre \& Suris, 2014; Oris et al., 2015), few interventional studies have included parents/caregivers of adolescents with any CC (Shilling et al., 2013), and fewer still with NF1. A pilot interventional study has tested the feasibility of an online chat-room support group for parents of children with NF1, which appeared to have potential as an efficacious method of providing support to parents (Martin et al., 2017). Published peer-support interventions that have included parents have aimed at creating a community of parents (Ainbinder et al., 1998; Law, King, Stewart, \& King, 2001; Solomon, Pistrang, \& Barker, 2001), allowed parents to share their experience as caregivers (Ainbinder et al., 1998; Kerr \& McIntosh, 2000; Kingsnorth, Gall, Beayni, \& Rigby, 2011), or to learn from the experiences of others (Ainbinder et al., 1998; Kingsnorth et al., 2011). However, these have largely included parents of children not adolescents (Martin et al., 2017), and those with disabilities, not somatic diseases. In particular, research has shown that social-emotional needs of youth with NF1 and their families can be addressed through internet-delivered interventions (Martin et al., 2014). Although it is crucial to provide parents with a support system and resources to help them in caring for their sick child, there is a dearth of peer support interventions for parents of adolescents with a CC (Shilling et al., 2013) or programs that involve them as 'transition experts' (Kingsnorth et al., 2011). Promoting peer support for parents can improve their own sense of well-being, 
which can, in turn, positively influence their child's health outcomes and illness management (Pardeck \& Pardeck, 1990). Peer support through social networking has been shown to have positive impacts on health behavior change (Laranjo et al., 2015). Despite recognition of the need for peer support, parents of children with a CC often have little extra time to participate in an intervention, particularly when it requires travelling to meet others (Akre et al., 2015).

Recognizing both the needs and the logistical limitations of parents and caregivers living with and caring for an adolescent with a CC, Children's at Home (C@H) was developed as a dedicated social networking site that offers a safe space to develop a community of shared experience. C@H combines the all-hours accessibility of social media with the proven efficacy of visual illness narratives created by young people with CCs. Video Intervention/Prevention Assessment (VIA) has been using patient-centered visual illness narratives for research and patient and parent education for more than two decades, with medical conditions ranging from asthma (Rich, Lamola, Amory, \& Schneider, 2000) to diabetes (Buchbinder et al., 2005) to obesity (Rich, Huecker, \& Ludwig, 2001). VIA collects data in the form of participant-generated "video diaries" of living with a medical condition (Chalfen \& Rich, 2004; Patashnick \& Rich, 2005; M. Rich, Lamola, Gordon, \& Chalfen, 2000; M Rich \& Patashnick, 2002). VIA visual narratives have revealed the hitherto unseen realities of living with CCs to clinicians and have allowed patients and families to learn from others who have faced similar challenges. VIA visual illness narratives have not only improved understanding of how CCs are managed outside the clinical setting, but the process of self-expression in documenting the illness experience has been demonstrated to improve patients' disease-specific quality of life (Rich, Lamola, \& Woods, 2006). Although previously VIA had only been used with child and adolescent patients who involved family and friends in documenting their illness experiences, we hypothesized that creating visual narratives and posting them on a social media site dedicated to parents of adolescents with CCs would reduce isolation and provide 
them with an opportunity for self-expression, empowerment, and crowdsourcing of ideas and concerns.

Social Learning Theory (SLT) (Bandura, 1986; Glanz \& Bishop, 2010) served as the theoretical framework for this study. We anticipated that parents would learn from each other's experience through peer-topeer education as they watched other parents' visual narratives, acquire new skills and behaviors in caring for their child with NF1, and receive emotional support from those who understood their experience. Indeed, social media have been shown to have an important impact on behavior, not only live modeling and media influence (Trujillo, Suarez, Lema, \& Londono, 2015) among adolescents and young adults (Qiu, Lin, Leung, \& Tov, 2012), but also among adults (Laranjo et al., 2015).

The purpose of this pilot study was to evaluate the feasibility and effect of $\mathrm{C} @ \mathrm{H}$, a secure social media site where parents of adolescents with NF1 could share visual narratives of their experiences.

\section{METHODS}

\section{Participants}

Parents and caregivers of adolescents (13-18 years) with NF1 (N=46) were invited to participate in C@H by email or in person by a clinician (NU) from the Boston Children's Hospital (BCH) Multidisciplinary Neurofibromatosis Program. Two email reminders were sent 1 and 2 months later to non-respondents; recruitment took a total of 3 months. This study was approved by the $\mathrm{BCH}$ Committee on Clinical Investigation (IRB). Parents interested in taking part were invited to contact the Project Coordinator (PC). At time of enrollment, the PC explained the study, obtained informed consent, and conducted a baseline semi-structured interview (TO) (Appendix). All participants were parents of children cared for in the BCH NF program and were enrolled in the study consecutively.

\section{Procedures}


The C@H study included 2 phases (Figure). Phase 1 consisted of parents creating VIA visual narratives of their experiences parenting and caring for an adolescent with NF1.

Parents were loaned a mini-tripod and phone adaptor so they could use their own smartphones to record their visual narratives. If a smartphone with video capacity was not available, a loaner was provided. Participants were instructed to make short ( $<5$ minute) videos about their experiences. Parents were provided 3 types of topic suggestions: (1) Daily talk suggestions, questions such as "How do you get along with your child with NF1?"; (2) Disease-specific suggestions, such as "Talk about your worries about having an adolescent with NF1"; (3) Interview questions for the parent to ask the adolescent with NF1, spouse, friend, or other person who knows them well, such as "Do you think I treat my child differently because s/he has NF1?". Participants uploaded their videos to their computer and used the $\mathrm{BCH}$ secure, protected File Transfer Protocol to send them to a private workspace created for each participant behind the hospital's firewall. The PC regularly provided technical assistance, feedback, and encouragement to the parent participants.

After creation of the visual narratives, participants completed a second semi-structured interview (T1) evaluating Phase 1 of the project (Appendix).

During Phase 2, parents interacted on C@H, a secure, medically-moderated social media website dedicated to parents of adolescents with NF1. The C@H website was created on Drupal (www.drupal.org) by a professional web developer and $\mathrm{BCH}$ watermarks were added to each video for security and ownership of information. The PC moderated and managed the website, edited videos for length and by content (long videos that touched on several subjects were edited into multiple videos with 1 video per subject); cleaning up head and tail of videos to remove wordless starting and stopping of the smartphone video; adjusting the audio levels and brightness for best quality playback; and removing identifiers (names, address, locations, etc. to make them anonymous). The PC and physician monitored specific videos for any possible incorrect or misleading medical information. Narratives 
mainly addressed the psychosocial difficulties of having an adolescent with NF1. Each video was assigned a unique title, short description, and a maximum of 6 tags based on the subjects addressed by visual narratives (e.g. "School concerns", “My child's social life”). All videos were uploaded on the C@H site, along with a separate "Welcome" video from the NF1 clinician. Parents were invited to view and interact with videos by posting comments, engaging in discussions, presenting new ideas, and uploading new visual narratives in response to what they saw. To access the website while ensuring security and confidentiality, participants created an anonymous username that was approved by the PC and created an initial two-password login. All posts were reviewed by the PC within 48 hours of receipt before editing and uploading to $\mathrm{C} @ \mathrm{H}$. Incoming comments, discussions, and videos were reviewed for content and edited by subject and for brevity before posting. Six videos were created by the NF1 clinician, explaining 6 different general NF1 topics such as "What is a neurofibroma?" and "What are learning issues typical of NF1?". Two topical health education videos were posted each week in conjunction with a gentle email reminder to participants to utilize the site. After interacting on $\mathrm{C} @ \mathrm{H}$, parents were asked to answer the semi-structured interview for the last time (T2) to evaluate their participation (Appendix). A \$10 Visa gift card was given at end of T0 (after the baseline information was collected, just before starting phase 1), $\$ 20$ at end of T1 and $\$ 20$ at end of T2.

To assess feasibility of the intervention, the PC conducted evaluations by phone or in-person in the form of semi-structured interviews (see Appendix). Those participants who could not schedule interviews answered the interview questions by email. At T0, participants were asked 19 open-ended questions regarding their use of social media, perceptions of NF1, prior interactions with other parents of children with NF1 in person or online, use of online tools to manage NF1, and expectations for C@H. At T1, participants were asked 11 open-ended questions evaluating their likes/dislikes about making visual narratives and suggestions for improvement. At T2, participants were asked 19 open-ended questions evaluating their use of $\mathrm{C@H}$, including: if and how it helped connect with others, likes/dislikes and feelings about the site, ease of use, what would improve their experience, and wishes for the future 
regarding NF1 online support. Interviews lasted between 45 minutes and 90 minutes. Responses were collected in the form of verbatim notes taken during interviews or text from participants' email responses.

\section{Data Analysis}

PC's interview notes or participants' written responses from T0, T1 and T2 were analyzed 1) across participants at each time point, and 2) within participants, before and after each phase of the intervention to determine positive or negative impressions, as well as barriers to use the C@H site according to their expectations (at T0) and their experience (at T1 and T2). Qualitative content analysis (Cho \& Lee, 2014) was used to analyze emerging themes to illustrate how parents of adolescents with NF1 were experiencing C@H. All data were coded in Excel by the first author (CA) in order to identify emerging themes in participants' opinions and perceptions at each phase of the intervention, the problems they encountered and how they resolved them. A total of 167 codes were created and independently reviewed and compared (CA and JP) to reach consensus. Theoretical sampling took place once the core variable of feeling isolated was defined. Constant comparative process was used until theoretical saturation was reached and further analysis was discontinued.

\section{RESULTS}

\section{TO semi-structured interviews}

Seventeen mothers of adolescent patients with NF1 enrolled in the study. Baseline demographic data, parents' use of social media and knowledge of others with NF1 are summarized in the Table.

Some parents found it difficult to talk about NF1 because of general lack of awareness, while others wanted to talk about it as much as possible to raise consciousness and understanding of the condition. All reported high interest in participating in a secure website to interact with peers who cared for an adolescent with NF1 because they felt lonely, desired a common place to share about their child's 
disease, and wanted an opportunity to talk to others with similar experiences about issues specific to NF1. What parents hoped to gain from C@H was: to know others were enduring similar problems ("Comfort of knowing that I can get accurate information from people who are going through the same thing"; "I hope to [...] compare notes for purposes of being on the same boat and sharing experiences"); to break the isolation and connect with other families who understood the struggles ("Connecting, seeing that I'm not alone dealing with some issues, find and give tips and advice"; "not feel so isolated"); to receive information from others going through similar experiences ("Hear insights from other parents. Share what I've been through with [my son] to help others. We are in the dark, we don't know; and it's more than just the medical aspects, it the whole social aspects"); to give and receive support and advice ("Support and to be able to support others"; "Find and give tips and advice"), especially when confronted with health issues or transition periods ("Middle school being a difficult time for many children, it is even harder for him with NF and cognitive issues"); and to develop friends for their children with NF1 who were struggling socially ("Connections for myself and for [my son]"; "To connect to other families and make friends for [my son]."). When asked what they would post about, the most important topics included: education/academic issues, social life, current health, how NF1 affects the family, and concerns about the future, mainly regarding their child's professional and social/romantic life.

Most participants felt that the site should have reliable medical oversight to guarantee medical accuracy and avoid the kind of misinformation that is common online. They did not feel that the oversight needed to be provided by a medical doctor, but someone who has solid knowledge of NF1, its specific challenges, and associated disorders. Some mentioned that they would like medical doctors to learn from C@H, particularly situations that arise outside of the clinical setting. Seven participants felt that medical issues were already discussed in consultations, and considered connections among parents of other children with NF1 addressing psychosocial problems to be more important than medical oversight.

Phase 1 
Over 6 months, 13 participants created 145 videos (1-21 videos per person; 0.5-7.2 minutes in length). Of the 17 enrolled parents, 4 did not complete VIA narratives due to lack of time.

\section{T1 semi-structured interviews}

At the end of Phase 1, the 13 remaining participants completed T1 semi-structured interviews.

Participants reported various levels of enjoyment creating visual narratives. Some reported that it felt like keeping a diary; they found it easy to speak to the camera and took pleasure in sharing their life experiences while exploring this form of communication. For others, creating videos felt awkward and unnatural; they were uncomfortable expressing themselves on camera and watching themselves, although this feeling decreased as they became more confident with the process and less cameraconscious.

What participants liked the most about recording their life on camera included: having the possibility to address topics not usually discussed in their family ("It brought up topics in my family that we don't discuss typically"); a forum to express their feelings and fears associated with having an adolescent with NF1 ("It allowed me to express my fears of having children with NF1") and how it affected them and their families ("It reminded me of how NF1 has affected our family"); the opportunity to speak about their journey since the NF1 diagnosis ("Made me realize how much NF1 has become part of all of our lives, and although the worry will always been there it has become "normal" for us and isn't as scary"; "It allowed me to speak candidly about the journey we have been through since finding out our son has NF1"); a space separate from daily life to reflect on living with NF1 ("Videos taught me how to reflect back, about parenting; listening to others speak helped me grow through reflections. Take a step back and reflect on whether I'm doing this correctly") ; an aid to realize how much NF1 had become a normal but challenging part of their lives ("I realize how much our son's NF1 really does affect my daily life"; "I often forget that my daughter has NF1, has challenges and they need to be taken into account"); the convenience of being able to record their experiences and thoughts anywhere ("Definitely get an 
understanding any time you want, access any time of the day from your own home"); the accessibility of a quick and easy way to tell a story ("There is not always time to go to event and stuff, so here it was easy to just go online anytime, from home"); and the enjoyment of including their child in the process ("The project has allowed my daughter with NF1 and me to talk more about it. [...] My daughter started asking more questions about her NF since participating"). What they least liked included: watching themselves on video and adding something else to their busy schedules.

Feedback regarding technical aspects focused on issues related to uploading their videos to the secure workspace; some had trouble, while others found it easy and straightforward.

\section{Phase 2}

The PC uploaded 145 videos onto C@H and created 115 tags (1-6 tags/video, ranging from 2\% with 1 tag to $41 \%$ with 6 tags; each tag was attached to $1-29$ videos). Examples of tags were "School transitions ", "My child's social life", "How NF affects my child", "Handling emotions", or "Family talk about NF1". The website was operational for 7 weeks; 10 participants engaged, 3 never logged on. Participants posted a total of 38 comments (1-8 comments/participant) and 18 new videos (1-7 videos/participant), with a mean of 2 logins/week/parent (self-reported). New videos addressed both new topics and responses to previous videos.

\section{T2 semi-structured interviews}

After interacting on C@H, the 10 participants completed T2 semi-structured interviews. All participants reported that they had never used or encountered a website like $\mathrm{C} @ \mathrm{H}$ and this was the first time they had connected with other parents of children with NF1. Some participants had previously engaged in online forums and on Facebook regarding NF1, but they strongly valued that C@H provided "real faces" and emotions of other parents sharing experiences. They felt that $\mathrm{C} @ \mathrm{H}$ provided them a sense of normalcy and a strong therapeutic effect. 
Seeing other parents of adolescents with NF1 online broke the isolation by sharing struggles ("If you are struggling with something but there is nobody listening or to share with, it is very lonely and isolated. Sharing is a very good thing."; "It is a comforting feeling that you are not alone, other folks who share what you are going through"), made them feel heard and understood ("IIt] felt like I was just as in-tuned with my kid as other parents, felt good because we are all on the same page"), gave them a sense of relief to talk about NF1 without having to explain it ("Most people who know me don't know that my children have NF1 because it's too complicated to explain, I don't even disclose the info at school. Except very close friends. So it was beneficial to connect to others for the first time without having to explain"), reassured them to see other families struggling with the same issues ("I learned that we all have similar concerns and fears"), and put their lives into perspective. Moreover, $\mathrm{C} @ \mathrm{H}$ enabled parents to address non-medical issues of NF1 that they never discussed with doctors ("[It was useful to] share about the non-medical perspective: we go to doctors all the time and they take care of all the medical aspects, but here we could address the non-medical ones."; "I learned things that I never really mentioned to doctors before because I thought it was [my son's] personality, I didn't think it was related to NF1"), and allowed them to discover individual versus NF1-related traits in their children ("Some things about [my son] I thought it was a him-thing [...]. Then I noticed that other boys were like him on the site. I was shocked that it was NF1- related").

Several parents described learning more about themselves by using $\mathrm{C} @ \mathrm{H}$. It pushed them out of their comfort zone to open up to others ("I was more open to sharing information and open up about myself. I don't like even having a picture taken, so this pushed me out of my comfort zone and helped me open $u p^{\prime \prime}$ ) and encouraged them to process and articulate feelings as they might in a diary ("What I learned: articulating feelings, like a diary. That was a good self-discipline to have to do in this department, to process things I was going through during that time. Very therapeutic."). Many questions required selfreflection; seeing themselves on video had an effect of taking a step back to reflect on parenting an adolescent with special needs. 
Most parents reported learning about associations between NF1 and chronic fatigue and NF1-related social difficulties that they previously believed to be personality-related adolescent issues. In addition, they reported learning how to better cope with NF1. They learned from others' narratives what they had not learned from books ("Parents with different issues was so interesting to hear. There is no book on this, hearing other people's perspectives, not just the theoretical books, but getting the first person memoires, getting a view in other people lives. Getting closer through people narratives"), they picked up tips on how to do what is best for their sick child, learned from others' successes ("Learning that [my son] is so similar to other NF kids and seeing how the parents handle it made me feel better [...] because they are concerned about the same as me"; "I saw similar difficulties, age issues, small successes we have in common"), and, after watching family interactions in visual narratives, understood how other families cope with NF1 in tangible ways ("[Seeing other parents with children with NF online] was great, they looked like the normal average family, it shows how NF1 becomes part of your life, everybody goes on with their lives").

Half of the parents reported being happy to talk more about NF1 because it brought up new issues, while the other half did not want NF1 to take over their lives. Whether or not they liked to talk about NF1, all parents who used C@H reported feeling better prepared to advocate with teachers, extended family members, and clinicians. They knew how to better answer questions, were able to explain their child's issues and concerns in simpler words, and felt legitimized by other parents feeling the same ("Another piece in my arsenal of expertise is good, for example: knowing more how to talk with teachers. [...] I know what to answer. [...] others' feelings carries the power of hearing others! Very powerful! And they are feelings, nobody can contest feelings").

What parents liked best about C@H: decreased isolation ("It made me feel that I am not the only parent of a child with NF"), being part of a supportive network ("It is comforting to know this community exists"; "It gave me the feeling that the others were going through the same, I felt the commiseration, 
and wanted to talk to them some more"; "When you have a rare disease, you are isolated, there are no support group"), accessing a wealth of experiential knowledge ("It was interesting to realize that they were like me, same experiences. I can relate to what I heard"; "I liked best the wealth of knowledge that it provided and used it as a resource that I didn't have before: to better understand, to see how other folks lived, how their environment was different from mine with a child with NF"), having confidence in state-of-the-art medical oversight ("The links would be medically accurate - can't always trust google, but here it was accurate information"), seeing other kids similar to theirs ("Il liked best] seeing people, seeing the kids. Not only seeing the other parents out there but also seeing the other kids"), and seeing other parents handle related challenges ("I learned that we all have similar concerns and fears"). What parents liked least: technological issues, being reminded that the disease was real, time constraints, and producing videos about themselves due to shyness.

Although all reported that C@H was easy to use, participants gave a few suggestions for improvement: add the capability to search for videos by person, streamline technical difficulties, create the possibility of connecting with other parents through Skype, and develop a 'Frequently Asked Questions' section. They considered C@H such a valuable 24/7 resource of information and strategies for living with and managing NF1 that they would like to see it continue on after the pilot was complete. Examples of future use were: to have $\mathrm{C} @ \mathrm{H}$ as a resource when new challenges occur (e.g. college graduation), to maintain connections with other parents, and to serve as an ongoing resource when needed.

\section{DISCUSSION}

Overall, Children's at Home for parents of adolescents with NF1 reached its primary goal of decreasing the isolation felt by parents by allowing them to connect and build a community of shared experience.

Results from T0 demonstrated a strong desire to connect with other parents of children with NF1 that had been identified in previous studies of adolescents with CC (Akre \& Suris, 2014). Findings from T1 
showed positive effects from creating VIA visual narratives. Although effectiveness of VIA has already been assessed with adolescents with other CCs (M. Rich, Lamola, Amory, et al., 2000; M. Rich et al., 2006), this is the first time it was applied to parents of affected children and to NF1 as a disease model. The therapeutic potential of VIA found among adolescent patients (Rich, Lamola \& Woods, 2006) was recapitulated in parents of adolescents with NF1 as they strongly valued the diary opportunity to tell about and reflect on their often complex lives with NF1. Results from T2 revealed that parents experienced relief to finally have the opportunity to encounter other parents with similar challenges and to share their experiences with NF1 without having to explain it. These results confirm the value of support groups for these parents and families (Tonsgard, 2006). Parents were comforted by the capability of visual narratives to put faces on those living with NF1 and to share others' emotions. Parenting an adolescent with a CC often comes with a sense of being different from parents of healthy children (Shilling et al., 2013); the visual narratives of others like them gave these parents a chance to recognize what is normal for them and their adolescent (Patterson \& Blum, 1996).

C@H allowed many participants to connect with other parents of affected children for the first time. A sense of isolation experienced by parents of adolescents with CC has been shown in previous European studies (Akre et al., 2015; Akre \& Suris, 2014). However, it might be reinforced in the United States by the Health Insurance Portability and Accountability Act of 1996 (HIPAA). The goal of HIPAA is to protect all individually identifiable health information in any form or media. A major and likely unintended consequence of HIPAA is that it contributes to patients' and parents' isolation by making it more difficult to meet others who share the same condition. This could contribute to the success of a trusted and secure social media website dedicated to those who share an illness experience, where parents can finally encounter peers and build a community of care in privacy and safety. Important questions regarding ethics, privacy and confidentiality have been raised about using social media in medicine and health care (Grajales, Sheps, Ho, Novak-Lauscher, \& Eysenbach, 2014). To optimize available technology that can promote public health while avoiding breaches in patient privacy, social media should be used 
wisely and prudently (Ventola, 2014). Possible reasons for not being part of open online social support platforms: a lack of trust that personal information and images will be respected and protected, lack of medical moderation and oversight, lack of trust in the platform, text-based communication of sensitive issues are more difficult than video narratives, and/or lack of control about who is included in the group (i.e. not necessarily peers).

Many participants reported having searched online about NF1, but were directed to misleading or unreliable information; T2 results showed that participants strongly valued that C@H was reliably moderated. Parents felt that the site moderator did not necessarily need to be a doctor, but it was most important that they provided trustworthy, state-of-the-art knowledge regarding NF1. Given this feedback, it seems safe to conclude that C@H could be expanded to a broader pool of participants while maintaining security (private, invitation only, multi-password login, watermarks, anonymity, firewall protection) and safety (medical moderation by a specialist). In stark contrast, open social media sites such as Facebook actually own all submitted content, which they can access and exploit, negating the confidential and secure nature that their "private" groups imply.

C@H offered a place where participants could discuss non-medical aspects of NF1 that they rarely had the opportunity to discuss in clinical settings, such as social functioning and worries for the future. The fact that such issues were not brought up during clinical visits might be linked to our finding that participants often viewed these issues as irrelevant to the medical care of NF1. Seeing other parents' narratives on C@H raised disease awareness, as some participants heard reports of other adolescents' NF1-related traits that they thought were personality-related in their child. Although these parents have been dealing with NF1 for many years, these misconceptions may have resulted from their isolation and limiting discussions with their child's doctor to medical aspects of NF1. These findings reveal a need to discuss psychosocial aspects of NF1 during consultations. As VIA has informed the medical community (Chalfen \& Rich, 2004) and identified key aspects of CCs not detected with standard clinical tools (Rich, 
Lamola \& Gordon, 2000), C@H could be used in the future as a teaching tool, to better inform health professionals regarding associated and indirect consequences of CCs.

The positive impact of the C@H intervention on parents is consistent with other peer-support interventions that have reduced isolation, loneliness and guilt (Ainbinder et al., 1998; Law et al., 2001; Solomon et al., 2001); created a shared social identity with other parents, an identity which cannot be fully understood by anyone not in a similar situation (Ainbinder et al., 1998; Kerr \& Mclntosh, 2000; Kingsnorth et al., 2011); allowed learning from the expertise and experience of other parents (Ainbinder et al., 1998; Kingsnorth et al., 2011); underscored the importance of mutuality, where providing support is as important as receiving it (Ainbinder et al., 1998); and provided parents a new awareness of how far they had come in their own journey since their child's diagnosis (Sullivan-Bolyai \& Lee, 2011). Still, these prior interventions have largely included parents of children, not adolescents, with disabilities or handicaps, not somatic diseases. C@H seems to be among the first to assess effects of supportive interventions for parents of adolescents with CCs and is the first such intervention for parents of adolescents with NF1.

The main strength of $\mathrm{C@H}$ was to help diminish the isolation felt by some parents of adolescents with NF1 by bringing online peer support directly into their homes. These parents were geographically isolated, but were finally able to "meet" others living similar experiences. A social network that brought together parents who cared for a CC, C@H allowed new relationships to develop by facilitating previously unavailable interactions (Coiera, 2013), generating homophily, the tendency of people to associate and bond with those who resemble them (McPherson, Smith-Lovin, \& Cook, 2001). Because it is accessed online, C@H has the potential for broader applicability and dissemination in a way that is lacking in traditional support interventions. C@H lessens the need to miss work and school to travel to receive specialized, face-to-face care and reduces health disparities since online tools are easily accessible by all (Ritterband \& Palermo, 2009). As demonstrated in prior studies using the VIA 
methodology with adolescents (M. Rich, Lamola, Amory, et al., 2000; M. Rich et al., 2002), producing videos was compared to keeping a diary, creating an opportunity to stop and reflect on the effects of the illness and thus taking a step back. Both producing visual narratives and watching those of others' proved beneficial as participants were able to share perspectives, challenges, and solutions, crowdsourcing knowledge as well as reassurance in seeing others going through similar experiences.

\section{Study Limitations}

Despite the overall success of $\mathrm{C@H}$, limitations exist. First, contrary to what has been shown with young people (M. Rich, Lamola, Gordon, et al., 2000), some parents were initially less comfortable using a camera in the "selfie" mode. However, once they overcame their initial resistance, even the most uncomfortable described positive aspects of pushing out of their comfort zone and into self-reflection. Second, this pilot study had a small sample size and several drop-outs, mostly due to lack of time. Despite their strong need for contact with peers, parents of children with CC have busy and stressful lives (Cousino \& Hazen, 2013), making the time investment necessary for this project difficult. To address the technical and time challenges faced by these participants, C@H will develop a smartphonebased mobile video app that allows users to create and instantly upload videos from anywhere at any time, just as millions of users do using commercial social media. This will address the most significant challenge to participants that resulted in our relatively high attrition rate. These challenges will be lessened by the all-in-one $\mathrm{C} @ H$ smartphone app. The fact that the majority of parents who enrolled completed the full study, stressed for time and energy as they were, indicates that parents really need and will invest the effort to obtain this support. Significant attrition is one of the fundamental characteristics and methodological challenges in the evaluation of eHealth applications (Eysenbach, 2005). Research has shown that for social media to work as an intervention, they need to fulfill needs that are not being met in offline lives (McLaughlin et al., 2012); those parents participating in C@H might well be those who did not find social support in other areas of their lives. 
Lastly, although all parents were invited to participate, only mothers formally engaged in the project.

Initially, this gender difference appeared to be of concern, but most mothers considered C@H a family project and eight of them included their child's father in their visual narratives. Nonetheless, incentives should be offered to encourage fathers to engage with C@H formally as well, since they have been shown to have a similar desire to connect with other parents and many offer different concerns regarding their child with a CC (Akre \& Suris, 2014; Goldstein, Akre, Belanger, \& Suris, 2013).

Given the number of visual narratives that parents created, the lengthy period they spent participating in the intervention, and the positive participants' reactions, C@H for parents of adolescents with NF1 appears to be a feasible intervention.

\section{Research recommendations}

Three key recommendations stem from the results of this pilot study. First, the technical complexity required of C@H participants should be streamlined into a smartphone application, so that participants can record and immediately upload their visual narratives "on the go". Second, given the relief parents felt from connecting and sharing, often for the first time, with other parents of adolescents with NF1, innovative applications of social media dedicated to caregivers should be developed for patients and families with other chronic conditions, offering peer-to-peer support and a $24 / 7$ informational resource exchange. Finally, future research can explore the value of separate, parallel C@H social media sites for patients with a CC and their parents/caregivers that can leverage the distancing effect of social media to improve communication and understanding between patients and their supportive families. Participants on each site can choose to share posts just with their peers or with the parallel site as well, thus showing and telling aspects of their experience that are too difficult or "hot" to discuss with their parents or children face-to-face. This "semi-permeable membrane" between parallel social media sites can allow both parents and children to observe sensitive, but important issues and concerns that can then be corrected by the other party. Caring relationships between young people with CCs and their caregivers 
are complex and often emotion-laden, so the "cooling effect" of discovering their own behaviors onscreen can allow problems to be corrected rather than ignored to avoid blame or shame. The C@H pilot demonstrated that secure social media sites dedicated to those who share an illness experience can be helpful not only to the patients but to those who do the labor-and time-intensive $24 / 7$ care that many of them need. 
Table : Demographics/Parent experiences

\begin{tabular}{|c|c|}
\hline \multicolumn{2}{|l|}{ Demographics/Parent experiences - Baseline T0 results $(\mathrm{N}=17)$} \\
\hline Child's gender & 8 females \\
\hline Child's age (median) & 15 years $(13-18)$ \\
\hline Prior social media experience & $15 / 17$ \\
\hline $\begin{array}{l}\text { Prior social media experience related to NF1 } \\
\text { (mainly closed NF1 groups on Facebook) }\end{array}$ & $9 / 17$ \\
\hline Experience with social media to meet and share with other parents of children with NF1 & $7 / 17$ \\
\hline Only at time of diagnosis & 4 \\
\hline Feel uncomfortable about reliability of information & 6 \\
\hline Found medically-moderated websites for parents & 0 \\
\hline \multicolumn{2}{|l|}{ Knows others with NF1 } \\
\hline No & 6 \\
\hline Has met one or two persons by chance (e.g. "my hair-dresser's son") & 7 \\
\hline Has met other people through NF1 camps or patient-family organization & 4 \\
\hline
\end{tabular}

NF1 - Neurofibromatosis 1 
Figure: Flow Diagram of the intervention

\section{Enrollment}

\section{Allocation}

\section{Follow-Up}

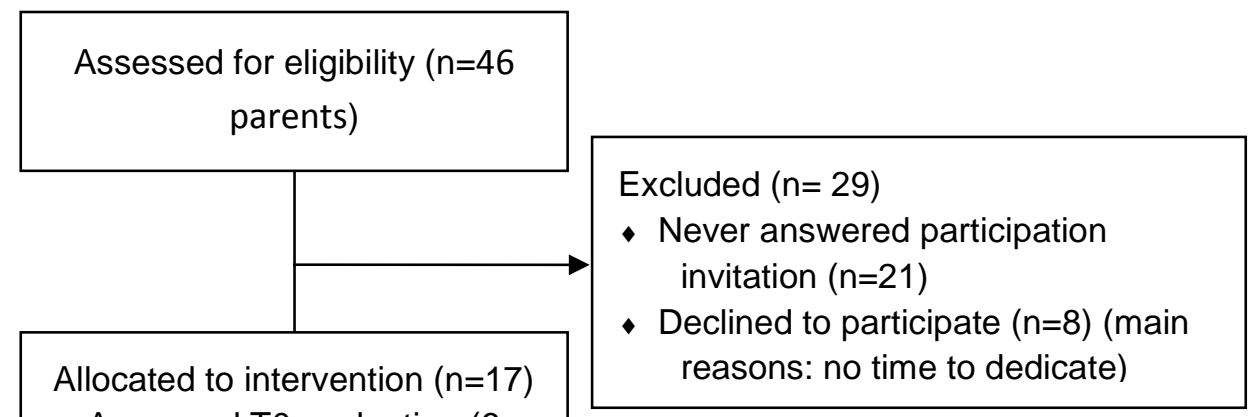

- Answered T0 evaluation (6 by phone, 2 in person, 9 by email)

Dropped-out $(n=4)$ (main reasons: no time to dedicate)

Phase 1: Creating visual narratives:

- 6 months

- $\mathrm{n}=13$

- 145 videos created

- Answered T1 evaluation ( $n=13)$ (All by email)

Never logged on $(n=3)$ (main reasons: no time to dedicate)

Phase 2: Interacting on C@H:

- 7 weeks

- $\mathrm{n}=10$

- 18 new videos created

- 38 comments posted

Answered T2 evaluation ( $\mathrm{n}=10)$
(8 by phone, 1 face to face, 1
by email)




\section{Acknowledgments}

This work was supported by the Children's Tumor Foundation Clinical Research Award to NU; and the Swiss National Science Foundation with a doc.mobility grant for a fellowship at the Boston Children's Hospital (grant number P1LAP3_151749) to CA. We would like to thank Kapil Patel for his work building the Children's at Home website and the participating families. 
Conflict of interest Author Akre Christina, Author Polvinen Julie, Author Ullrich Nicole J, and Author Rich Michael declare that they have no conflict of interest.

Human Studies and Informed Consent All procedures followed were in accordance with the ethical standards of the responsible committee on human experimentation (institutional and national) and with the Helsinki Declaration of 1975, as revised in 2000. Informed consent was obtained from all patients for being included in the study.

Animal studies No animal studies were carried out by the authors for this article. 


\section{References}

Ainbinder, J. G., Blanchard, L. W., Singer, G. H., Sullivan, M. E., Powers, L. K., Marquis, J. G., \& Santelli, B. (1998). A qualitative study of Parent to Parent support for parents of children with special needs. Consortium to evaluate Parent to Parent. J Pediatr Psychol, 23(2), 99-109.

Akre, C., Ramelet, A. S., Berchtold, A., \& Suris, J. C. (2015). Educational intervention for parents of adolescents with chronic illness: a pre-post test pilot study. Int J Adolesc Med Health, 27(3), 261269.

Akre, C., \& Suris, J. C. (2014). From controlling to letting go: what are the psychosocial needs of parents of adolescents with a chronic illness? Health Educ Res, 29(5), 764-772.

Bandura, A. (1986). Social Foundations of Thought and Action: A Social Cognitive Theory. NJ: PrenticeHall.

Barton, B., \& North, K. (2004). Social skills of children with neurofibromatosis type 1. Dev Med Child Neurol, 46(8), 553-563.

Benjamin, C. M., Colley, A., Donnai, D., Kingston, H., Harris, R., \& Kerzin-Storrar, L. (1993). Neurofibromatosis type 1 (NF1): knowledge, experience, and reproductive decisions of affected patients and families. J Med Genet, 30(7), 567-574.

Brehaut, J. C., Kohen, D. E., Garner, R. E., Miller, A. R., Lach, L. M., Klassen, A. F., \& Rosenbaum, P. L. (2009). Health among caregivers of children with health problems: findings from a Canadian population-based study. Am J Public Health, 99(7), 1254-1262.

Brehaut, J. C., Kohen, D. E., Raina, P., Walter, S. D., Russell, D. J., Swinton, M., Rosenbaum, P. (2004). The health of primary caregivers of children with cerebral palsy: how does it compare with that of other Canadian caregivers? Pediatrics, 114(2), e182-191.

Buchbinder, M. H., Detzer, M. J., Welsch, R. L., Christiano, A. S., Patashnick, J. L., \& Rich, M. (2005). Assessing adolescents with insulin-dependent diabetes mellitus: a multiple perspective pilot study using visual illness narratives and interviews. J Adolesc Health, 36(1), 71 e79-13.

Chalfen, R., \& Rich, M. (2004). Applying visual research: patients teaching nphysicians about asthma through visual illness narratives. Visual Anthropology Review, 20(1), 17-30.

Cho, J. Y., \& Lee, E. (2014). Reducing Confusion abouth Grounded Theory and Qualitative Content Analysis: Similarities and Differences. The Qualitative Report, 19(32), 1-20.

Coiera, E. (2013). Social networks, social media, and social diseases. BMJ, 346, $\mathrm{f} 3007$.

Coude, F. X., Mignot, C., Lyonnet, S., \& Munnich, A. (2006). Academic Impairment is the Most Frequent Complication of Neurofibromatosis Type-1 (NF1) in Children. Behav Genet, 1-5.

Cousino, M. K., \& Hazen, R. A. (2013). Parenting stress among caregivers of children with chronic illness: a systematic review. J Pediatr Psychol, 38(8), 809-828.

Dilts, C. V., Carey, J. C., Kircher, J. C., Hoffman, R. O., Creel, D., Ward, K., Leonard, C. O. (1996). Children and adolescents with neurofibromatosis 1: a behavioral phenotype. J Dev Behav Pediatr, 17(4), 229-239.

Eysenbach, G. (2005). The law of attrition. J Med Internet Res, 7(1), e11.

Ferner, R. E. (2007). Neurofibromatosis 1 and neurofibromatosis 2: a twenty first century perspective. Lancet Neurol, 6(4), 340-351.

Frey, K. S., Greenberg, M. T., \& Fewell, R. R. (1989). Stress and coping among parents of handicapped children: a multidimensional approach. Am J Ment Retard, 94(3), 240-249.

Garwood, M. M., Bernacki, J. M., Fine, K. M., Hainsworth, K. R., Davies, W. H., \& Klein-Tasman, B. P. (2012). Physical, cognitive, and psychosocial predictors of functional disability and healthrelated quality of life in adolescents with neurofibromatosis-1. Pain Res Treat, 2012.

Glanz, K., \& Bishop, D. B. (2010). The role of behavioral science theory in development and implementation of public health interventions. Annu Rev Public Health, 31, 399-418. 
Goldstein, H., Akre, C., Belanger, R. E., \& Suris, J. C. (2013). Detached, distraught or discerning? Fathers of adolescents with chronic illness: a review of the literature. Int J Adolesc Med Health, 25(2), 109-117.

Graf, A., Landolt, M. A., Mori, A. C., \& Boltshauser, E. (2006). Quality of life and psychological adjustment in children and adolescents with neurofibromatosis type 1. J Pediatr, 149(3), 348-353.

Grajales, F. J., 3rd, Sheps, S., Ho, K., Novak-Lauscher, H., \& Eysenbach, G. (2014). Social media: a review and tutorial of applications in medicine and health care. J Med Internet Res, 16(2), e13.

Hyman, S. L., Arthur Shores, E., \& North, K. N. (2006). Learning disabilities in children with neurofibromatosis type 1: subtypes, cognitive profile, and attention-deficit-hyperactivity disorder. Dev Med Child Neurol, 48(12), 973-977.

Hyman, S. L., Shores, A., \& North, K. N. (2005). The nature and frequency of cognitive deficits in children with neurofibromatosis type 1. Neurology, 65(7), 1037-1044.

Johnson, N. S., Saal, H. M., Lovell, A. M., \& Schorry, E. K. (1999). Social and emotional problems in children with neurofibromatosis type 1: evidence and proposed interventions. J Pediatr, 134(6), 767-772.

Kerr, S. M., \& McIntosh, J. B. (2000). Coping when a child has a disability: exploring the impact of parentto-parent support. Child Care Health Dev, 26(4), 309-322.

Kingsnorth, S., Gall, C., Beayni, S., \& Rigby, P. (2011). Parents as transition experts? Qualitative findings from a pilot parent-led peer support group. Child Care Health Dev, 37(6), 833-840.

Laranjo, L., Arguel, A., Neves, A. L., Gallagher, A. M., Kaplan, R., Mortimer, N., Lau, A. Y. (2015). The influence of social networking sites on health behavior change: a systematic review and metaanalysis. J Am Med Inform Assoc, 22(1), 243-256.

Law, M., King, S., Stewart, D., \& King, G. (2001). The perceived effects of parent-led support groups for parents of children with disabilities. Phys Occup Ther Pediatr, 21(2-3), 29-48.

Levine, T. M., Materek, A., Abel, J., O'Donnell, M., \& Cutting, L. E. (2006). Cognitive profile of neurofibromatosis type 1. Semin Pediatr Neurol, 13(1), 8-20.

Martin, S., Gillespie, A., Wolters, P. L., \& Widemann, B. C. (2011) Experiences of families with a child, adolescent, or young adult with neurofibromatosis type 1 and plexiform neurofibroma evaluated for clinical trials participation at the National Cancer Institute. Contemp Clin Trials, 32(1), 10-15.

Martin, S., Roderick, M. C., Lockridge, R., Toledo-Tamula, M. A., Baldwin, A., Knight, P., \& Wolters, P. (2017). Feasibility and Preliminary Efficacy of an Internet Support Group for Parents of a Child with Neurofibromatosis Type 1: a Pilot Study. J Genet Couns, 26(3), 576-585.

Martin, S., Wolters, P., Baldwin, A., Gillespie, A., Dombi, E., Walker, K., \& Widemann, B. (2012). Socialemotional functioning of children and adolescents with neurofibromatosis type 1 and plexiform neurofibromas: relationships with cognitive, disease, and environmental variables. J Pediatr Psychol, 37(7), 713-724.

Martin, S., Wolters, P. L., Baldwin, A., Roderick, M. C., Toledo-Tamula, M. A., Gillespie, A., \& Widemann, B. (2014). Attitudes about internet support groups among adolescents and young adults with neurofibromatosis type 1 and their parents. J Genet Couns, 23(5), 796-804.

McKinney, B., \& Peterson, R. A. (1987). Predictors of stress in parents of developmentally disabled children. J Pediatr Psychol, 12(1), 133-150.

McLaughlin, M., Nam, Y., Gould, J., Pade, C., Meeske, K. A., Ruccione, K. S., \& Fulk, J. (2012). A videosharing social networking intervention for young adult cancer survivors. Computers in Human Behavior, 28, 631-641.

McPherson, M., Smith-Lovin, L., \& Cook, J. M. (2001). Birds of a feather: Homophily in social networks. Annu Rev Sociol, 27, 415-444. 
Oris, L., Seiffge-Krenke, I., Moons, P., Goubert, L., Rassart, J., Goossens, E., \& Luyckx, K. (2016). Parental and peer support in adolescents with a chronic condition: a typological approach and developmental implications. J Behav Med. 39(1):107-19

Pardeck, J. A., \& Pardeck, J. T. (1990). Family factors related to adolescent autonomy. Adolescence, 25(98), 311-319.

Patashnick, J., \& Rich, M. (2005). Researching human nexperience: Video Intervention/Prevention Assessment (VIA). Australasian Journal of Information Systems, 36(1), 71.e79-71.e13.

Patterson, J., \& Blum, R. W. (1996). Risk and resilience among children and youth with disabilities. Arch Pediatr Adolesc Med, 150(7), 692-698.

Qiu, L., Lin, H., Leung, A. K., \& Tov, W. (2012). Putting their best foot forward: emotional disclosure on Facebook. Cyberpsychol Behav Soc Netw, 15(10), 569-572.

Reiter-Purtill, J., Schorry, E. K., Lovell, A. M., Vannatta, K., Gerhardt, C. A., \& Noll, R. B. (2008). Parental Distress, Family Functioning, and Social Support in Families with and without a Child with Neurofibromatosis 1. Journal of Pediatric Psychology 33(4), 422-434.

Rich, M., Huecker, D., \& Ludwig, D. (2001). Obesity in the lives of children and adolescents: inquiry through patient-centered visual narratives. Pediatric Research, 49, 7A.

Rich, M., Lamola, S., Amory, C., \& Schneider, L. (2000). Asthma in life context: Video Intervention/Prevention Assessment (VIA). Pediatrics, 105(3 Pt 1), 469-477.

Rich, M., Lamola, S., Gordon, J., \& Chalfen, R. (2000). Video intervention/prevention assessment: a patient-centered methodology for understanding the adolescent illness experience. $J$ Adolesc Health, 27(3), 155-165.

Rich, M., Lamola, S., \& Woods, E. R. (2006). Effects of creating visual illness narratives on quality of life with asthma: a pilot intervention study. J Adolesc Health, 38(6), 748-752.

Rich, M., \& Patashnick, J. (2002). Narrative research with audiovisual data: Video Intervention/Preention Assessment (VIA) and NVivo. International Journal of Social Research and Methodology, 5(3), 245-261.

Rich, M., Patashnick, J., Huecker, D., \& Ludwig, D. (2002). Living with obesity: visual narratives of overweight adolescents. Journal of Adolescent Health, 30, 100.

Ritterband, L. M., \& Palermo, T. M. (2009). Introduction to the special issue: eHealth in pediatric psychology. J Pediatr Psychol, 34(5), 453-456.

Shilling, V., Morris, C., Thompson-Coon, J., Ukoumunne, O., Rogers, M., \& Logan, S. (2013). Peer support for parents of children with chronic disabling conditions: a systematic review of quantitative and qualitative studies. Dev Med Child Neurol, 55(7), 602-609.

Solomon, M., Pistrang, N., \& Barker, C. (2001). The benefits of mutual support groups for parents of children with disabilities. Am J Community Psychol, 29(1), 113-132.

Sullivan-Bolyai, S., \& Lee, M. M. (2011). Parent mentor perspectives on providing social support to empower parents. Diabetes Educ, 37(1), 35-43.

Tonsgard, J. H. (2006). Clinical manifestations and management of neurofibromatosis type 1. Semin Pediatr Neurol, 13(1), 2-7.

Trujillo, E., Suarez, D., Lema, M., \& Londono, A. (2015). How adolescents learn about risk perception and behavior in regards to alcohol use in light of social learning theory: a qualitative study in Bogota, Colombia. Int J Adolesc Med Health, 27(1), 3-9.

Ventola, C. L. (2014). Social media and health care professionals: benefits, risks, and best practices. $P T$, 39(7), 491-520. 